\title{
Yukawa particles in a confining potential
}

Cite as: J. Chem. Phys. 141, 014106 (2014); https://doi.org/10.1063/1.4885723

Submitted: 11 April 2014 . Accepted: 17 June 2014 . Published Online: 03 July 2014

Matheus Girotto, Alexandre P. dos Santos, Thiago Colla, and Yan Levin

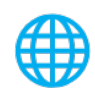

\section{ARTICLES YOU MAY BE INTERESTED IN}

Simulations of Coulomb systems with slab geometry using an efficient 3D Ewald summation method

The Journal of Chemical Physics 144, 144103 (2016); https://doi.org/10.1063/1.4945560 Ionic size effects on the Poisson-Boltzmann theory

The Journal of Chemical Physics 147, 014104 (2017); https://doi.org/10.1063/1.4990737

Charge neutrality breakdown in confined aqueous electrolytes: Theory and simulation The Journal of Chemical Physics 145, 094704 (2016); https://doi.org/10.1063/1.4962198

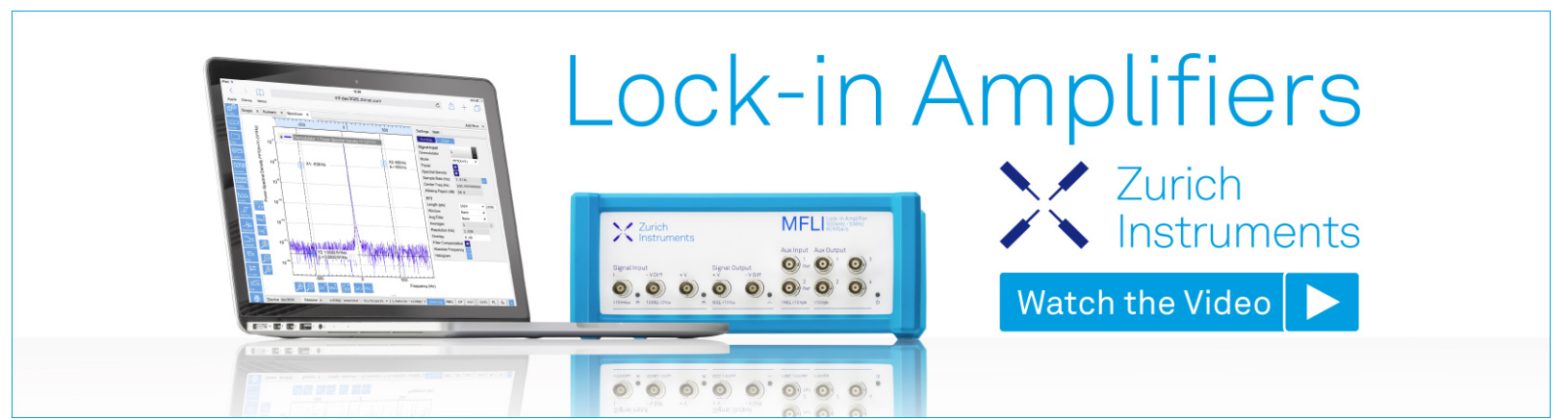




\title{
Yukawa particles in a confining potential
}

\author{
Matheus Girotto, ${ }^{1, a)}$ Alexandre P. dos Santos, ${ }^{2,3, b)}$ Thiago Colla, ${ }^{4, c)}$ and Yan Levin ${ }^{1, d)}$ \\ ${ }_{1}^{1}$ Instituto de Física, Universidade Federal do Rio Grande do Sul, Caixa Postal 15051, CEP 91501-970, \\ Porto Alegre, RS, Brazil \\ ${ }^{2}$ Departamento de Educação e Informação em Saúde, Universidade Federal de Ciências da Saúde de Porto \\ Alegre, 90050-170, Porto Alegre, RS, Brazil \\ ${ }^{3}$ Departamento de Física, Universidade Federal de Santa Catarina, 88040-900, Florianópolis, \\ Santa Catarina, Brazil \\ ${ }^{4}$ Faculty of Physics, University of Vienna, Boltzmanngasse 5, A-1090 Vienna, Austria
}

(Received 11 April 2014; accepted 17 June 2014; published online 3 July 2014)

We study the density distribution of repulsive Yukawa particles confined by an external potential. In the weak coupling limit, we show that the mean-field theory is able to accurately account for the particle distribution. In the strong coupling limit, the correlations between the particles become important and the mean-field theory fails. For strongly correlated systems, we construct a density functional theory which provides an excellent description of the particle distribution, without any adjustable parameters. (O) 2014 AIP Publishing LLC. [http://dx.doi.org/10.1063/1.4885723]

\section{INTRODUCTION}

The Yukawa potential is used to model interparticle interactions in plasmas, ${ }^{1,2}$ dusty plasmas, ${ }^{3-5}$ colloidal suspensions, ${ }^{6-8}$ and atomic physics. ${ }^{9,10}$ In soft-matter systems, the exponential screening of the effective potential arises from the positional correlations between the oppositely charged particles. ${ }^{11,12}$ Because of its great importance for various models, the thermodynamics of Yukawa systems has been a subject of extensive study. ${ }^{13-15}$ Most of the previous work, however, has been restricted to the homogeneous fluid or solid states. ${ }^{16-22}$ In this paper, we will investigate a gas of Yukawa particles confined by an external potential. Such situation arises, for example, when a colloidal system is acted on by the electromagnetic field produced by the laser tweezers. ${ }^{23-30}$ Without a loss of generality, in this paper, we will consider the external potential which has a one dimensional parabolic form

$$
W(z)=\frac{\alpha z^{2}}{2},
$$

where $\alpha$ is a measure of the trap strength. The theory developed below, however, can be applied to an arbitrary confining potential $W(x, y, z)$.

We will first show that in the weak-coupling limit (high temperatures), the density distribution of Yukawa gas is well described by the mean-field (MF) theory. ${ }^{31,32}$ In the strong coupling limit (low temperature), the positional correlations between the particles become important and the MF theory fails. ${ }^{33-35}$ In this case, we will construct a density functional theory (DFT) based on the hypernetted-chain (HNC) equation and the local density approximation (LDA) and will show that this theory accounts very accurately for the particle dis-

\footnotetext{
a)Electronic mail: matheus.girotto@ufrgs.br

b) Electronic mail: alexandreps@ufcspa.edu.br

c)Electronic mail: thiago.colla@ufrgs.br

d) Electronic mail: levin@if.ufrgs.br
}

tribution. All the theoretical results will be compared with the Monte Carlo (MC) simulations.

\section{MEAN-FIELD THEORY}

We study a system of $N$ particles interacting through a repulsive Yukawa potential

$$
V(r)=q G\left(\mathbf{r}_{1}, \mathbf{r}_{2}\right),
$$

where

$$
G\left(\mathbf{r}_{1}, \mathbf{r}_{2}\right)=q \frac{e^{-\lambda r}}{r}
$$

$r=\left|\mathbf{r}_{1}-\mathbf{r}_{2}\right|, \lambda$ is the typical inverse distance, and $q$ is the strength of the interaction potential. For colloidal systems, $q$ is

$$
q=\frac{Z e}{\sqrt{\epsilon_{w}}},
$$

where $Z e$ is the charge of colloidal particles, $e$ is the proton charge, $\epsilon_{w}$ is the dielectric constant of the medium, and $\lambda$ is the inverse Debye length which depends on the ionic strength inside the suspension. ${ }^{12}$

We first observe that $G\left(\mathbf{r}_{1}, \mathbf{r}_{2}\right)$ satisfies the Helmholtz equation

$$
\nabla^{2} G\left(\mathbf{r}, \mathbf{r}_{1}\right)-\lambda^{2} G\left(\mathbf{r}, \mathbf{r}_{1}\right)=-4 \pi q \delta\left(\mathbf{r}-\mathbf{r}_{1}\right) .
$$

Consider a Yukawa gas confined to a hyperstripe with periodic boundary conditions in the $x$ and $y$ directions and open in the $z$ direction. The solution of Eq. (5) for such system can be expressed as

$$
G\left(\mathbf{r}, \mathbf{r}_{1}\right)=\frac{2 \pi q}{L_{x} L_{y}} \sum_{m_{x}, m_{y}} e^{2 \pi i\left[\frac{m_{x}}{L_{x}}\left(x-x_{1}\right)+\frac{m_{y}}{L_{y}}\left(y-y_{1}\right)\right]} \frac{e^{-\gamma_{m}\left|z-z_{1}\right|}}{\gamma_{m}},
$$


where

$$
\gamma_{m}=\sqrt{\lambda^{2}+4 \pi^{2}\left(\frac{m_{x}^{2}}{L_{x}^{2}}+\frac{m_{y}^{2}}{L_{y}^{2}}\right)},
$$

and $-\infty<m_{x}<\infty$ and $-\infty<m_{y}<\infty$ are integers. $L_{x}$ and $L_{y}$ are the widths of the hyperstripe in the $x$ and $y$ directions, respectively.

In equilibrium, the distribution of confined particles is given by

$$
\rho(z)=A e^{-\beta \omega(z)},
$$

where $\beta=1 / k_{B} T, \omega(z)$ is the potential of mean force (PMF), and $A$ is the normalization constant. ${ }^{12}$ In the weak-coupling limit, the correlations between the particles can be neglected and the PMF can be approximated by $\omega(z)=q \phi(z)+W(z)$, where $\phi(z)$ is the Yukawa potential at position $z$. This constitutes a MF approximation for the particle distribution,

$$
\rho(z)=A e^{-\beta[q \phi(z)+W(z)]},
$$

where

$$
A=\frac{N}{L_{x} L_{y} \int_{-\infty}^{+\infty} d z e^{-\beta[q \phi(z)+W(z)]}} .
$$

The potential $\phi(z)$ can be expressed in terms of the Green's function, Eq. (6),

$$
\phi(z)=\int d \mathbf{r}^{\prime} \rho\left(z^{\prime}\right) G\left(\mathbf{r}, \mathbf{r}^{\prime}\right)
$$

Integrating over $x$ and $y$ coordinates, Eq. (11) simplifies to

$$
\phi(z)=\frac{2 \pi q A}{\lambda} \int_{-\infty}^{+\infty} d z^{\prime} e^{-\beta\left[q \phi\left(z^{\prime}\right)+W\left(z^{\prime}\right)\right]-\lambda\left|z^{\prime}-z\right|},
$$

which is an integral equation for the mean field potential. This equation can be solved numerically using Picard iteration.

To test the accuracy of the MF theory, we perform MC simulations. $N=100$ Yukawa particles are confined in a box of sides $L_{x}, L_{y}$, and $L_{z}$, with periodic boundary conditions in $x$ and $y$ directions. In the $z$ direction, the particles are constrained by an external potential $W(z)$. We set $L_{z}=200 \lambda^{-1}$. The periodic lengths are taken to be $L_{x}=L_{y}=35 \lambda^{-1}$, while the cutoff for the particle-particle interaction is set at $10 \lambda^{-1}$. In the Metropolis algorithm, a new configuration $n$ is constructed from an old configuration $o$ by a small displacement of a random particle. The new state is accepted with a probability $P=\min \left\{1, e^{-\beta\left(E_{n}-E_{o}\right)}\right\}$, where $E_{n}$ and $E_{o}$ are the energies of the new and the old configurations, respectively. If the movement is not accepted, the configuration $o$ is preserved and counted as a new state. The length of the displacement is adjusted during the simulation in order to obtain the acceptance rate of $50 \%$. The energy of the system used in the MC simulations is given by

$$
E=\sum_{i=1}^{N-1} \sum_{j=i+1}^{N} q G\left(\mathbf{r}_{i}, \mathbf{r}_{j}\right)+\sum_{i=1}^{N} W\left(z_{i}\right) .
$$

The averages are calculated using $10^{5}$ uncorrelated states, obtained after $10^{6} \mathrm{MC}$ steps for equilibration. To quantify the strength of the particle-particle interaction and the trapparticle interaction, it is convenient to define the following

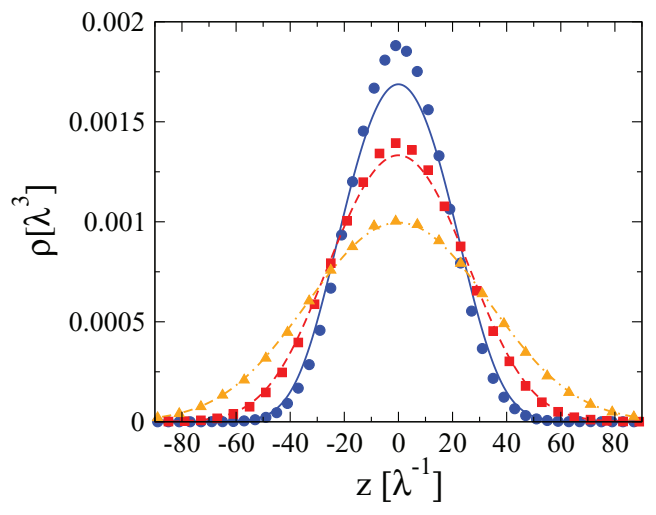

FIG. 1. Density profile of Yukawa gas with $\epsilon=40$ and $\chi=0.004$ (circles); $\epsilon=20$ and $\chi=0.002$ (squares); and $\epsilon=10$ and $\chi=0.001$ (triangles). The symbols represent $\mathrm{MC}$ simulation data, while the curves are the predictions of MF theory.

dimensionless parameters

$$
\epsilon=\frac{q^{2} \lambda}{k_{B} T} \quad \text { and } \quad \chi=\frac{\alpha}{k_{B} T \lambda^{2}} .
$$

We can now compare the solutions of the MF equations (9) and (12) with the results of MC simulations, see Fig. 1. For high temperatures - low values of $\epsilon$ - the MF theory accounts very well for the particle distribution observed in the MC simulations. On the other hand, in the strong coupling limit (low temperatures), the correlations between the particles become important and the MF theory starts to fail. Positional correlations between the particles lead to greater occupation of the low energy states than is predicted by the MF theory. ${ }^{12}$ This is similar to the process of overcharging observed in colloidal suspensions with multivalent ions. ${ }^{36-38}$

\section{DENSITY FUNCTIONAL THEORY}

The failure of the MF theory to properly account for the density distribution of a confined Yukawa gas is a consequence of strong positional correlations between the particles at low temperatures. To account for these correlations, we appeal to the DFT. The equilibrium particle distribution corresponds to the minimum of the Helmholtz free energy

$$
F[\rho(z)]=F^{e n t}+F^{i n t}+F^{c o r}
$$

subject to constraint

$$
\int d \mathbf{r} \rho(\mathbf{r})=N
$$

In Eq. (15), $F^{e n t}$ is the entropic contribution to the free energy, $F^{i n t}$ is the interaction part (which includes both the MF interaction and the interaction with the external potential), and $F^{c o r}$ is the correlational free energy. In general, the correlational free energy is a non-local function of density $\rho(z)$. For systems with hard-core interactions, this requires development of sophisticated weighted density approximations. ${ }^{39-43}$ For repulsive Yukawa particles, however, the density variation should be much smoother and we expect that a LDA for $F^{c o r}[\rho(z)]$ will be sufficiently accurate. LDA assumes that the system achieves a local thermodynamic equilibrium within 
a range smaller than the typical length scale of the system inhomogeneity. ${ }^{44,45}$ This condition is fulfilled provided that the density distribution does not vary dramatically. Since the density profiles resulting from the soft potential, Eq. (1), are smooth (see Fig. 1), we expect that the LDA will be sufficiently accurate in the present situation as long as $\epsilon$ and $\chi$ are not too large. Performing the minimization of the total free energy, we obtain the equilibrium particle density distribution, ${ }^{12}$

$$
\rho(z)=N \frac{e^{-\beta\left[q \phi(z)+W(z)+\mu^{c o r}[\rho(z)]\right]}}{L_{x} L_{y} \int_{-\infty}^{+\infty} d z e^{-\beta\left[q \phi(z)+W(z)+\mu^{c o r}[\rho(z)]\right]}},
$$

where the correlational chemical potential is

$$
\mu^{c o r}[\rho(z)]=\frac{\delta F^{c o r}}{\delta \rho(z)} .
$$

Within the LDA, $\mu^{\text {cor }}[\rho(z)]$ is calculated using the free energy of a homogeneous system

$$
\mu^{\operatorname{cor}}[\rho(z)]=\left.\frac{\partial f^{\operatorname{cor}}(\bar{\rho})}{\partial \bar{\rho}}\right|_{\bar{\rho}=\rho(z)},
$$

where $f^{c o r}$ is the correlational free energy density of a homogeneous Yukawa gas. When the correlations are negligible (high temperatures), $\mu^{\text {cor }}$ vanishes and the MF theory, Eq. (9), becomes exact.

To calculate the correlational chemical potential, we use the HNC equation. This equation is known to account well for the structural and thermodynamic properties of Yukawa-like systems. ${ }^{46,47}$ The HNC approximation is based on a closure relation

$$
h(\mathbf{r})=\ln [-\beta v(\mathbf{r})+h(\mathbf{r})-c(\mathbf{r})]-1,
$$

for the Ornstein-Zernike equation, where $h(\mathbf{r})$ is the pair correlation function, $c(\mathbf{r})$ is the direct correlation function, and $v(\mathbf{r})$ is the particle-particle interaction potential. In the Fourier space, the Ornstein-Zernike equation, for an isotropic system, takes a particularly simple form,

$$
h(k)=\frac{c(k)}{1-\rho c(k)} .
$$

This equation can be solved iteratively. First, we make an initial guess for the direct correlation function, $c_{0}(r)$. The Fourier transform of $c_{0}(r)$ is then inserted into Eq. (21), yielding a zero order approximation of $h_{0}(k)$. The inverse Fourier transformation provides $h_{0}(r)$. The closure relation, Eq. (20), allows us to calculate the next order direct correlation function, $c_{1}(r)$, etc. The process is repeated until convergence is achieved. ${ }^{45}$ To speed up the convergence, a method of $\mathrm{Ng}$ with six parameters is used for updating the $c(r)$ at each iteration step. $^{48}$

Within the HNC approximation, the excess (over the ideal gas) chemical potential ${ }^{45}$ is

$$
\beta \mu^{e x}=\frac{1}{2} \rho \int h(\mathbf{r})[h(\mathbf{r})-c(\mathbf{r})] d \mathbf{r}-\rho \int c(\mathbf{r}) d \mathbf{r} .
$$

In Fig. 2, we show that the Eq. (22) agrees perfectly with the chemical potential calculated using the MC simulations and Widom particle insertion algorithm. ${ }^{49}$

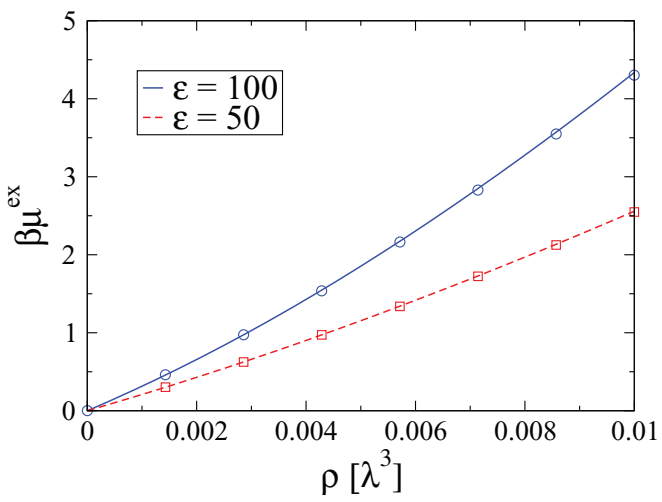

FIG. 2. The excess (over the ideal gas) chemical potential as a function of the particle concentration for $\epsilon=100$ and $\epsilon=50$. The symbols represent the simulation data, and the lines are calculated using the HNC equation and Eq. (22).

The excess chemical potential contains both the MF and the correlational contributions. The correlational chemical potential, $\mu^{\text {cor }}$, is calculated by subtracting from $\mu^{e x}$ the MF part

$$
\mu^{m f}=\frac{\partial F^{m f}}{\partial N}
$$

where the MF free energy of a homogeneous Yukawa gas is

$$
F^{m f}=\frac{q^{2}}{2} \rho^{2} \int d^{3} \mathbf{r} \int d^{3} \mathbf{r}^{\prime} \frac{e^{-\lambda\left|\mathbf{r}-\mathbf{r}^{\prime}\right|}}{\left|\mathbf{r}-\mathbf{r}^{\prime}\right|} .
$$
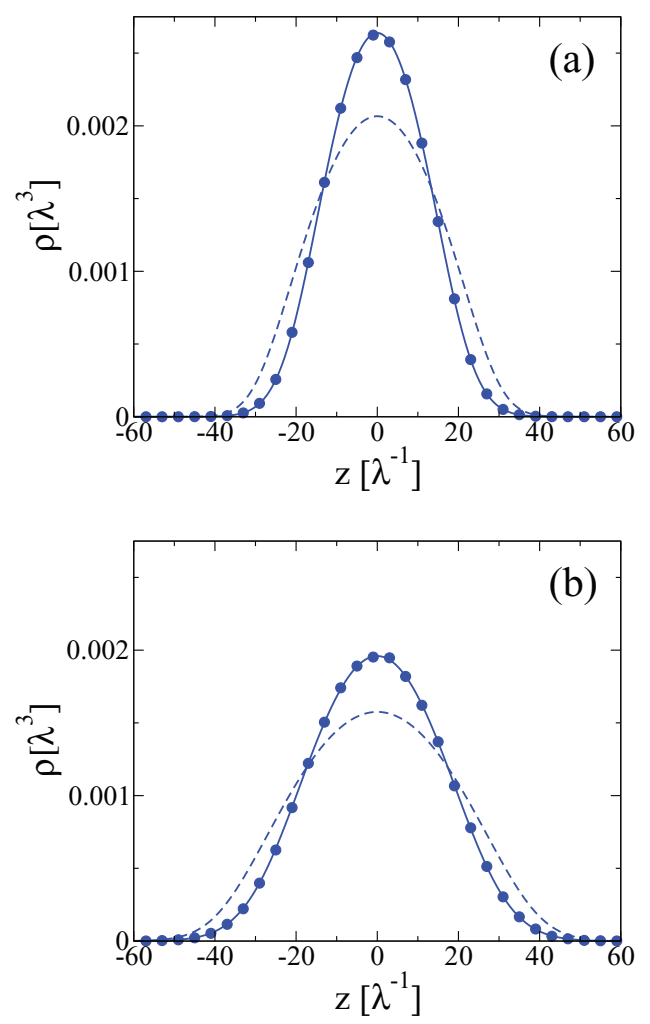

FIG. 3. Concentration profiles for $\epsilon=100$. The symbols represent MC simulation data, the solid curves represent the DFT, and the dashed curves the MF theory. The $\chi$ parameters are 0.01 and 0.005 , for (a) and (b), respectively. 

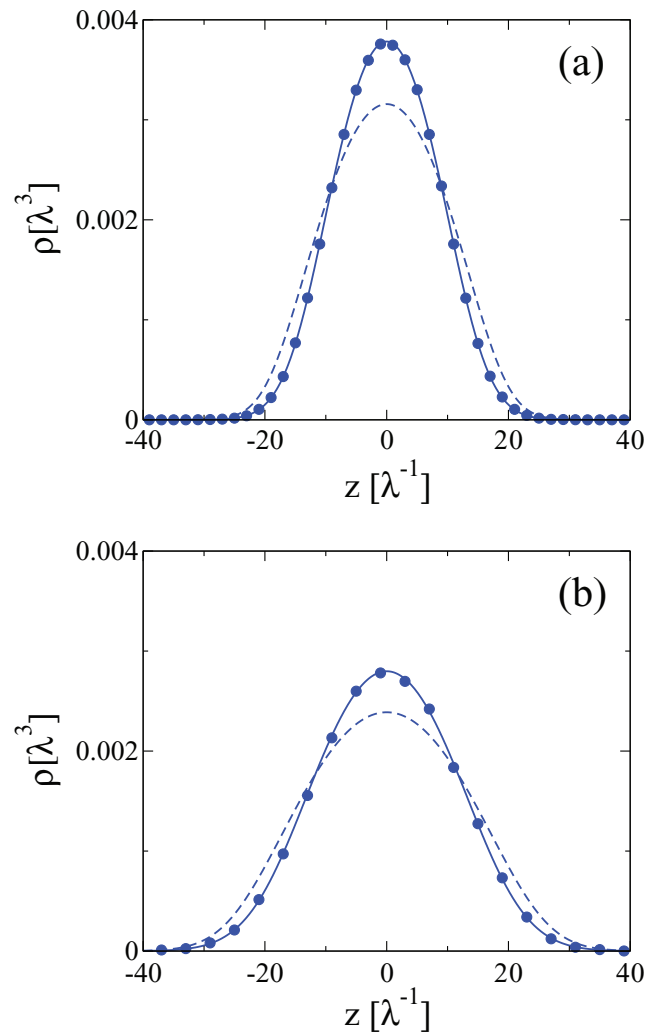

FIG. 4. Concentration profiles for $\epsilon=50$. The symbols represent MC simulation data, the solid curves represent the DFT, and the dashed curves the MF theory. The $\chi$ parameters are 0.02 and 0.01 , for (a) and (b), respectively.

Integrating Eq. (24) and then differentiating with respect to $N$, we obtain

$$
\beta \mu^{m f}=\frac{4 \pi \rho q^{2} \beta}{\lambda^{2}} .
$$

To calculate the density profile of an inhomogeneous Yukawa gas confined by an external potential, the system of equations (12) and (17), and the HNC equation must be solved simultaneously. In practice, to speed up the calculations, we first calculate the chemical potential of a homogeneous Yukawa system. The solution of the HNC equation shows that to a very high degree of accuracy the correlational

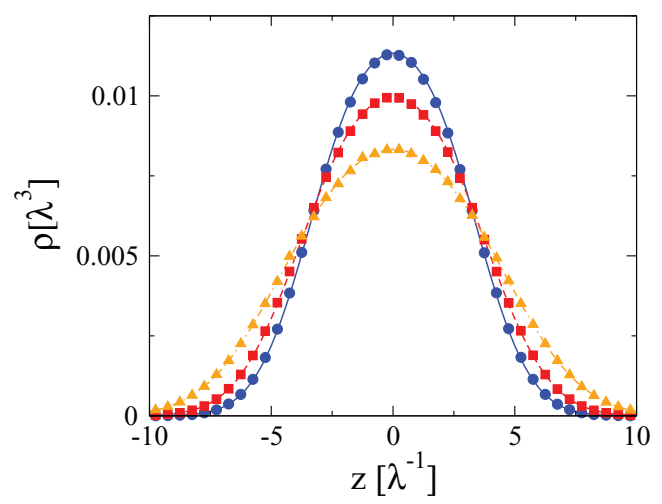

FIG. 5. Density profile of Yukawa gas with $\epsilon=20$ and $\chi=0.2$ (circles); $\chi=0.15$ (squares); and $\chi=0.1$ (triangles). The symbols represent MC simulation data, while the curves are the predictions of the theory.

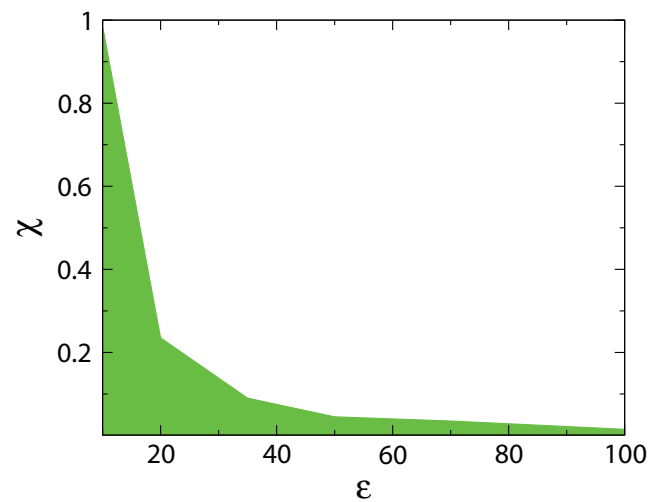

FIG. 6. The shadowed area shows the parameters $\chi$ and $\epsilon$ for which the self-consistent equations based on the LDA present converged solutions. The values of $N, L_{x}$, and $L_{y}$ are the same as previously.

chemical potential has a simple parabolic form $\beta \mu^{\text {cor }}=a \rho$ $+b \rho^{2}$. The HNC equation allows us to calculate the parameters $a$ and $b$ for various values of $\epsilon$. To speed up the numerical integration, we can then use the approximate form of the LDA approximation, $\beta \mu^{c o r}[\rho(z)]=a \rho(z)+b \rho^{2}(z)$, in Eq. (17). In Figs. 3 and 4, we compare the theoretically calculated density profiles obtained using HNC-LDA with the results of MC simulations. We see that, while the MF theory fails to account for the simulation results, the DFT based on the HNC equation and the LDA is able to provide an extremely accurate description of the particle distribution, without any adjustable parameters. Perhaps surprisingly, the theory remain very accurate even in the very strong coupling limit of $\epsilon=100$.

In Fig. 5, we show the evolution of the particle distribution as the strength of the confining potential $\chi$ increases. The agreement between the theory and the simulations remains excellent until the moment when the self-consistent equations fail to converge, indicating a breakdown of the LDA. The shaded region of Fig. 6 shows the parameter space in which the self-consistent equations of the LDA present a convergent solution. For $\epsilon$ and $\chi$ outside the shaded region, the correlations between the particles become sufficiently strong to result in a breakdown of the LDA.

\section{CONCLUSIONS}

We have studied a gas of Yukawa particles confined by an external potential. In the weak coupling limit, we have constructed a MF theory which allows us to accurately calculate the equilibrium particle density distribution inside a confining potential. In the strong coupling limit, the correlations between the particles become important and the MF theory fails. We show, however, that a DFT theory based on the HNC equation and a LDA approximation accounts perfectly for the observed particle distributions even in the limit of very strong interactions between the particles. The LDA, however, is shown to fail when the correlations between the particles become sufficiently strong to lead to a local ordering of the fluid. If the temperature is sufficiently low and the number of particles is sufficiently high, we expect that the Yukawa fluid will crystallize at the bottom of the potential. This 
crystallization transition, however, lies outside the range of validity of the present theory.

\section{ACKNOWLEDGMENTS}

This work was partially supported by the CNPq, INCTFCx, and by the US-AFOSR under the Grant No. FA9550-121-0438.

${ }^{1}$ M. C. Zammit, D. V. Fursa, and I. Bray, Phys. Rev. A 82, 052705 (2010).

${ }^{2}$ A. Basu, J. Phys. B: At. Mol. Opt. Phys. 43, 115202 (2010).

${ }^{3}$ H. Totsuji, T. Kishimoto, C. Totsuji, and T. Sasabe, Phys. Rev. E 58, 7831 (1998).

${ }^{4}$ V. E. Fortov, A. V. Ivlev, S. A. Khrapak, A. G. Khrapak, and G. E. Morfill, Phys. Rep. 421, 1 (2005).

${ }^{5}$ K. N. Dzhumagulova, T. S. Ramazanov, and R. U. Masheeva, Phys. Plasmas 20, 113702 (2013).

${ }^{6}$ E. J. Meijer and D. Frenkel, Phys. Rev. Lett. 67, 1110 (1991).

${ }^{7}$ M. Heinen, P. Holmqvist, A. J. Banchio, and G. Nägele, J. Chem. Phys. 134, 044532 (2011).

${ }^{8}$ M. N. van der Linden, A. van Blaaderen, and M. Dijkstra, J. Chem. Phys. 138, 114903 (2013).

${ }^{9}$ L. G. Jiao and Y. K. Ho, Int. J. Quantum Chem. 113, 2569 (2013).

${ }^{10}$ O. Certik and P. Winkler, Int. J. Quantum Chem. 113, 2012 (2013).

${ }^{11}$ C. N. Likos, Phys. Rep. 348, 267 (2001).

${ }^{12}$ Y. Levin, Rep. Prog. Phys. 65, 1577 (2002).

${ }^{13}$ S. Hamaguchi, R. T. Farouki, and D. H. E. Dubin, Phys. Rev. E 56, 4671 (1997)

${ }^{14}$ M. H. J. Hagen and D. Frenkel, J. Chem. Phys. 101, 4093 (1994).

${ }^{15}$ E. Scholl-Paschinger, N. E. Valadez-Perez, A. L. Benavides, and R. Castaneda-Priego, J. Chem. Phys. 139, 184902 (2013).

${ }^{16}$ M. O. Robbins, K. Kremer, and G. S. Grest, J. Chem. Phys. 88, 3286 (1988).

${ }^{17}$ E. J. Meijer and D. Frenkel, J. Chem. Phys. 94, 2269 (1991).

${ }^{18}$ H. Löwen, T. Palberg, and R. Simon, Phys. Rev. Lett. 70, 1557 (1993).

${ }^{19}$ T. Palberg, W. Mönch, F. Bitzer, T. Bellini, and R. Piazza, Phys. Rev. Lett. 74, 4555 (1995).

${ }^{20}$ M. J. Stevens and M. O. Robbins, J. Chem. Phys. 98, 2319 (1993).

${ }^{21}$ R. S. Hoy and M. O. Robbins, Phys. Rev. E 69, 056103 (2004).
${ }^{22}$ J. Gapinski, G. Nägele, and A. Patkowski, J. Chem. Phys. 136, 024507 (2012).

${ }^{23}$ J. C. Crocker and D. G. Grier, Phys. Rev. Lett. 77, 1897 (1996).

${ }^{24}$ J. C. Crocker, J. Chem. Phys. 106, 2837 (1997).

${ }^{25}$ D. G. Grier, Curr. Opin. Colloid Interface Sci. 2, 264 (1997).

${ }^{26}$ E. R. Dufresne and D. G. Grier, Rev. Sci. Instrum. 69, 1974 (1998).

${ }^{27}$ B. H. Lin, J. Yu, and S. A. Rice, Phys. Rev. E 62, 3909 (2000).

${ }^{28}$ H. Löwen, J. Phys.: Condens. Matter 13, R415 (2001).

${ }^{29}$ A. Resnick, J. Colloid Interface Sci. 262, 55 (2003).

${ }^{30}$ M. M. Elmahdy, C. Gutsche, and F. Kremer, J. Phys. Chem. C 114, 19452 (2010).

${ }^{31}$ Y. Levin and R. Pakter, Phys. Rev. Lett. 107, 088901 (2011).

${ }^{32}$ M. Girotto, A. P. dos Santos, and Y. Levin, Phys. Rev. E 88, 032118 (2013).

${ }^{33}$ R. van Roij and J. P. Hansen, Phys. Rev. Lett. 79, 3082 (1997).

${ }^{34}$ S. Alexander, P. M. Chaikin, P. Grant, G. J. Morales, P. Pincus, and D. Hone, J. Chem. Phys. 80, 5776 (1984).

${ }^{35}$ Y. Levin, M. C. Barbosa, and A. Diehl, Europhys. Lett. 41, 123 (1998).

${ }^{36}$ A. Y. Grosber, T. T. Nguyen, and B. I. Shklovskii, Rev. Mod. Phys. 74, 329 (2002).

${ }^{37}$ S. Pianegonda, M. C. Barbosa, and Y. Levin, Europhys. Lett. 71, 831 (2005).

${ }^{38}$ A. P. dos Santos, A. Diehl, and Y. Levin, J. Chem. Phys. 132, 104105 (2010).

${ }^{39}$ Y. Rosenfeld, Phys. Rev. Lett. 63, 980 (1989).

${ }^{40}$ Y. Rosenfeld, M. Schmidt, H. Löwen, and P. Tarazona, J. Phys.: Condens. Matter 8, L577 (1996).

${ }^{41}$ R. Evans, Lecture Notes at 3rd Warsaw School of Statistical Physics (Warsaw University Press, Kazimierz Dolny, 2009), pp. 43-85.

${ }^{42}$ R. Roth, J. Phys.: Condens. Matter 22, 063102 (2010).

${ }^{43}$ D. Frydel and Y. Levin, J. Chem. Phys. 138, 174901 (2013).

${ }^{44}$ R. Evans, Adv. Phys. 28, 143 (1979).

${ }^{45}$ J. P. Hansen and I. R. McDonald, Theory of Simple Liquids, 3rd ed. (Academic, London, 2006).

${ }^{46}$ P. Attard, "Electrolytes and the Electric Double Layer," in Advances in Chemical Physics, edited by I. Prigogine and S. A. Rice (John Wiley \& Sons, Inc., Hoboken, NJ, 2007), Vol. 92.

${ }^{47}$ T. E. Colla, A. P. dos Santos, and Y. Levin, J. Chem. Phys. 136, 194103 (2012).

${ }^{48}$ K. Ng, J. Chem. Phys. 61, 2680 (1974).

${ }^{49}$ D. Frenkel and B. Smit, Understanding Molecular Simulation: From Algorithms to Applications, Computational Science Series (Elsevier Science, 2001). 